\title{
STABILIZATION OF ADIABATIC COUETTE-POISEUILLE FLOW BY TEMPERATURE DEPENDENT VISCOSITY*
}

\author{
BY
}

MOSES A. BOUDOURIDES (Democritus University of Thrace, Xanthi, Greece)

AND

NICOLAS CH. CHARALAMBAKIS (Aristotle University of Thessaloniki, Thessaloniki, Greece)

\begin{abstract}
We consider the adiabatic plane Couette-Poiseuille flow of a viscous incompressible fluid between two parallel planes driven by the shearing of the upper plane and a pressure gradient. The viscosity is assumed to depend on the temperature in an appropriate way to insure that every classical solution of the governing equations is asymptotically attracted by the steady Couette-Poiseuille flow profile with the temperature increasing unlimitedly. The proof is based on a priori estimates, obtained by the help of certain identities for solutions of the governing equations.
\end{abstract}

1. Consider an incompressible Newtonian viscous fluid in the region between two parallel rigid plates located at $x=0$ and $x=1$. If, perpendicularly to the $x$ direction, the upper plate moves with velocity $V$ and a pressure gradient $-P>0$ is applied, the resulting flow is usually named (plane) Couette-Poiseuille.

Assuming that the viscosity of the fluid depends on its temperature and that the flow is adiabatic (i.e., no thermal diffusion), we are interested in determining the asymptotic behavior of the velocity $v$, shear stress $\sigma$ and temperature $\theta$ of the fluid. Actually, these fields are coupled in a system of a parabolic and a hyperbolic partial differential equation governing the flow. So, the asymptotic behavior of the solutions depends on the outcome of the competition between the stabilizing effect of parabolicity and the destabilizing effect of hyperbolicity.

Indeed, the evolution of $v, \sigma$, and $\theta$ is ruled by the following momentum and energy balances (cf. [1])

$$
\left.\begin{array}{l}
v_{t}=\sigma_{x}+P \\
\theta_{t}=\sigma v_{x}
\end{array}\right\} \quad 0<x<1,0<t<\infty
$$

under the constitutive assumption

$$
\sigma=\mu(\theta) v_{x}
$$

where the viscosity coefficient $\mu$ is assumed to be a decreasing positive function of temperature (as typically for liquids) of the form

$$
\mu(\theta)=\bar{\mu}+\theta^{-\gamma}
$$

for a positive exponent $\gamma$ and a positive limiting viscosity $\bar{\mu}$ as $\theta \rightarrow \infty$.

*Received February 6, 1989. 
The above system is accompanied with the following boundary and initial conditions

$$
\begin{gathered}
v(0, t)=0, \quad v(1, t)=V, \quad 0 \leq t<\infty, \\
v(x, 0)=v_{0}(x), \quad \theta(x, 0)=\theta_{0}(x), \quad 0 \leq x \leq 1 .
\end{gathered}
$$

As energy is constantly pumped into the fluid (by the boundary shearing and the pressure gradient) and the flow is adiabatic, the temperature will keep rising and asymptotically $\theta \rightarrow \infty$. Then the viscosity will approach the constant asymptotic value $\bar{\mu}$ and it is reasonable to expect that $v$ will asymptotically approach the steady solution of $(1.1),(1.5)$ with $\mu=\bar{\mu}$, which has the following profile

$$
\bar{v}=\frac{P}{2 \bar{\mu}} x(1-x)+V x .
$$

In fact, in our next main result, we verify this conjecture (under the condition $0<$ $\gamma<1$ ), thus, establishing that the steady Couette-Poiseuille flow (1.7) is the unique asymptotically stable solution of the system (1.1), (1.2), (1.5), (1.6).

Theorem. Assume that $\bar{\mu}>0,0<\gamma<1, v_{0} \in W^{2,2}(0,1), \theta_{0} \in W^{1,2}(0,1), v_{0}(0)=0$, $v_{0}(1)=V, \theta_{0}(x)>0,0 \leq x \leq 1$. Then there is a unique solution $v(x, t), \theta(x, t)$ of (1.1), (1.2), (1.5), (1.6) on $[0,1] \times[0, \infty)$ and, as $t \rightarrow \infty$, uniformly in $x \in[0,1]$,

$$
\begin{gathered}
\sigma(x, t)=-P x+O\left(t^{-\gamma-1}\right), \\
v(x, t)=\frac{P}{2 \bar{\mu}} x(1-x)+V x+O\left(t^{-\gamma-1}\right), \\
v_{t}(x, t)=O\left(t^{-\gamma-1}\right), \\
\theta(x, t)=O(t) .
\end{gathered}
$$

The case $P=0, V=1$ (i.e., plane Couette flow) was treated in [2] for $\bar{\mu}=0$ and in [3] for general $\mu(\theta)$.

2. Proof of theorem. Let us assume that $v(x, t), \theta(x, t)$ is a solution of $(1.1)$, (1.2), (1.5), (1.6) on $[0,1] \times[0, \infty)$ such that $v, v_{x}, v_{t}, v_{x x}, \theta, \theta_{x} \in C\left([0, \infty) ; L^{2}(0,1)\right)$, $v_{x t} \in C\left((0, \infty) ; L^{2}(0,1)\right)$ and $v_{t t} \in L_{\text {loc }}^{2}\left((0, \infty) ; L^{2}(0,1)\right)$. Then we proceed to establish a priori estimates, which will lead to the proof of the theorem. In what follows, $K$ will stand for a generic positive constant, which can be estimated from above solely in terms of $\bar{\mu}, \gamma, P, V$, and the norms of $v_{0}, \theta_{0}$.

We rewrite $(1.1),(1.2)$ as

$$
\begin{gathered}
v_{t}=\left(\left(\bar{\mu}+\theta^{-\gamma^{\prime}}\right) v_{x}\right)_{x}+P, \\
\theta_{t}=\left(\bar{\mu}+\theta^{-\gamma^{\prime}}\right) v_{x}^{2} .
\end{gathered}
$$

Multiplying (2.2) by $\mu(\theta)$ (given in (1.4)) we can easily deduce that $\theta$ is increasing in $t$ and

$$
K^{-1} \leq \mu \leq K, \quad 0<x<1, \quad 0 \leq t<\infty .
$$


Multiplying (2.1) by $v_{t}$ and integrating we obtain

$$
\begin{gathered}
\int_{0}^{t}\left\|v_{t}\right\|^{2} d \tau+\frac{1}{2} \int_{0}^{t} \int_{0}^{1} \mu v_{x}^{2} d x+\frac{\gamma}{2} \int_{0}^{t} \int_{0}^{1} \theta^{-\gamma-1} \mu v_{x}^{4} d x d \tau \\
=P \int_{0}^{1} v d x-P \int_{0}^{1} v_{0} d x-\frac{1}{2} \int_{0}^{1} \mu_{0} v_{0 x}^{2} d x,
\end{gathered}
$$

where $\|\cdot\|$ denotes the norm of $L^{2}(0,1)$. Using the Poincare inequality and (2.3)

$$
P \int_{0}^{1} v d x \leq|P||| v_{x}||<\varepsilon \int_{0}^{1} \mu v_{x}^{2} d x+K, \quad 0<\varepsilon<\frac{1}{2},
$$

and so (2.4) implies the first a priori estimate

$$
\int_{0}^{t}\left\|v_{t}\right\|^{2} d \tau+\left\|v_{x}\right\|^{2} \leq K, \quad 0 \leq t<\infty .
$$

Next we evaluate $\|\sigma\|$. Because of (2.3), the Cauchy-Schwarz inequality and (1.5)

$$
\|\sigma\|^{2}=\int_{0}^{1} \mu^{2} v_{x}^{2} d x \geq K^{-1}
$$

and because of $(2.3)$ and $(2.5)$

$$
\|\sigma\|^{2} \leq K\left\|v_{x}\right\|^{2} \leq K
$$

i.e., we have shown that

$$
K^{-1} \leq\|\sigma\| \leq K, \quad 0 \leq t<\infty .
$$

Multiplying (2.2) by $\mu$ we get the following useful relation

$$
\begin{aligned}
\left(\bar{\mu} \theta+\frac{1}{1-\gamma} \theta^{1-\gamma^{\prime}}\right)_{t}=\sigma^{2}= & \int_{0}^{1} \sigma^{2} d y+2 \int_{0}^{1} \int_{y}^{x} \sigma(\xi, t) v_{t}(\xi, t) d \xi d y \\
& -2 P \int_{0}^{1} \int_{y}^{x} \sigma(\xi, t) d \xi d y .
\end{aligned}
$$

Integrating (2.7) over $[0, t]$ and using (2.5), (2.6), and the Cauchy-Schwarz inequality, we obtain the following a priori estimate for $\theta$

$$
K^{-1} t \leq \theta \leq K t, \quad 0 \leq x \leq 1, \quad 1 \leq t<\infty,
$$

which shows (1.11).

Our next step is to evaluate $\left\|v_{t}\right\|$. For this purpose, first we differentiate (2.1) with respect to $t$ to get

$$
v_{t t}=\left(\mu v_{x t}-\gamma \theta^{-\gamma-1} \mu v_{x}^{3}\right)_{x} .
$$

Multiplying (2.9) by $v_{t}$ and integrating over [0,1], we obtain

$$
\frac{1}{2} \frac{d}{d t}\left\|v_{t}\right\|^{2}+\int_{0}^{1} \mu v_{x t}^{2} d x=\gamma \int_{0}^{1} \theta^{-\gamma-1} \mu v_{x}^{3} v_{x t} d x
$$

i.e., applying the Poincaré and the Cauchy-Schwarz inequality,

$$
\frac{d}{d t}\left\|v_{t}\right\|^{2}+K^{-1}\left\|v_{t}\right\|^{2} \leq K \int_{0}^{1} \theta^{-2 \gamma^{-2}} \mu v_{x}^{6} d x
$$


Using (2.8), (2.6), (2.3) in the last relation, we get

$$
\begin{aligned}
\frac{d}{d t}\left\|v_{t}\right\|^{2}+K^{-1}\left\|v_{t}\right\|^{2} & \leq K t^{-2 \gamma-2}\left\|\sigma^{3}\right\|^{2} \\
& \leq K t^{-2 \gamma-2}\left\{\max _{[0.1]} \sigma^{2}\right\} .
\end{aligned}
$$

However, applying the Cauchy-Schwarz and the Poincare inequality in (2.7), we get, after (2.6) and (2.3),

$$
\sigma^{2} \leq K\|\sigma\|^{2}+2\|\sigma\|\left\|v_{t}\right\| \leq K+K\left\|v_{x t}\right\| \leq K+\varepsilon\left\|v_{x t}\right\|^{2},
$$

for a sufficiently small $\varepsilon>0$, and so (2.10) becomes

$$
\frac{d}{d t}\left\|v_{t}\right\|^{2}+K^{-1}\left\|v_{t}\right\|^{2} \leq K t^{-2 ;-2} \text {. }
$$

Integration of the last differential inequality, after taking into account (2.5), results

$$
\left\|v_{t}\right\|=O\left(t^{-\gamma-1}\right), \quad \text { as } t \rightarrow \infty .
$$

Consequently, (2.6) and (2.11) applied in (2.7) yield

$$
\sigma=O(1), \quad 0 \leq x \leq 1, \quad t \rightarrow \infty,
$$

and (2.12) together with (2.3) applied in (1.3) yield

$$
v_{x}=O(1), \quad 0 \leq x \leq 1, \quad t \rightarrow \infty .
$$

Now we proceed to estimate $\left\|v_{x t}\right\|$. Multiplying (2.9) by $t^{2} v_{t}$ and integrating, we get

$$
\frac{1}{2} t^{2}\left\|v_{t}\right\|^{2}-\int_{0}^{t} \int_{0}^{1} \tau v_{t}^{2} d x d \tau+\int_{0}^{t} \int_{0}^{1} \tau^{2} \mu v_{x t}^{2} d x d \tau=\gamma \int_{0}^{t} \int_{0}^{1} \tau^{2} \theta^{-r^{-1}} \mu v_{x}^{3} v_{x t} d x d \tau,
$$

from which, with the help of (2.11), (2.8), (2.13), (2.3) and the Cauchy-Schwarz inequality, we obtain

$$
\int_{0}^{t} \int_{0}^{1} \tau^{2} \mu v_{x t}^{2} d x d \tau \leq K t^{-2 \gamma+1}, \quad \text { as } t \rightarrow \infty .
$$

Next, we multiply (2.9) by $t^{3} v_{t t}$ and integrate to get, after some integrations by parts

$$
\begin{aligned}
\int_{0}^{t} \int_{0}^{1} \tau^{3} v_{t t}^{2} d x d \tau+\frac{1}{2} t^{3} \int_{0}^{1} \mu v_{x t}^{2} d x \\
=\frac{3}{2} \int_{0}^{t} \int_{0}^{1} \tau^{2} \mu v_{x t}^{2} d x d \tau-\frac{7 \gamma}{2} \int_{0}^{t} \int_{0}^{1} \tau^{3} \theta^{-\gamma^{-1}} \mu v_{x}^{2} v_{x t}^{2} d x d \tau \\
\quad+\gamma t^{3} \int_{0}^{1} \theta^{-\gamma-1} \mu v_{x}^{3} v_{x t} d x-3 \gamma \int_{0}^{t} \int_{0}^{1} \tau^{2} \theta^{-\gamma^{-1}} \mu v_{x}^{3} v_{x t} d x d \tau \\
\quad+\gamma(\gamma+1) \int_{0}^{t} \int_{0}^{1} \tau^{3} \theta^{-\gamma^{-2}} \mu^{2} v_{x}^{5} v_{x t} d x d \tau+\gamma^{2} \int_{0}^{t} \int_{0}^{1} \tau^{3} \theta^{-2 \gamma^{-2}} \mu v_{x}^{5} v_{x t} d x d \tau,
\end{aligned}
$$

which yields, in combination with (2.14), (2.13), (2.8), and (2.3),

$$
\left\|v_{x t}\right\|=O\left(t^{-r^{-1}}\right), \quad \text { as } t \rightarrow \infty .
$$


The last estimate and (1.5) imply (1.10). This combined with (1.1), yields (1.8) by an integration. Finally, integrating (1.8) and using (1.4), we obtain (1.9).

Once the above a priori estimates have been derived, the existence of a unique globally defined solution of $(1.1),(1.2),(1.5),(1.6)$ can be established by a standard procedure. Actually, having a uniform estimate for $\theta$ (by (2.8)) and $v$ (by the maximum principle), one can apply the Leray-Schauder fixed point theorem (as in [4]) to show local existence on a maximal time interval and then use (2.13) and (2.8) to show that this solution cannot escape in finite time.

\section{REFERENCES}

[1] G. K. Batchelor, An Introduction to Fluid Dynamics, Cambridge University Press, London, 1970

[2] C. M. Dafermos, Stabilizing effects of dissipation, Equadiff 82, Lecture Notes in Mathematics, vol. 1017, H. W. Knobloch and K. Schmitt (eds.), Springer-Verlag, Berlin, 1983, pp. 140-147

[3] C. M. Dafermos and L. Hsiao, Adiabatic shearing of incompressible fluids with temperature dependent viscosity, Quart. Appl. Math. 41, 45-58 (1983)

[4] A. E. Tzavaras. Effect of thermal softening in shearing of strain-rate dependent materials, Arch. Rational Mech. Anal. 99, 349-374 (1987) 